\title{
Reanimación facial en Síndrome de Möbius completo mediante transferencia en un solo tiempo de gracilis libre bilateral
}

\section{One-stage facial reanimation with bilateral free gracilis muscle transfer in complete Möbius Syndrome}

\author{
Alexandro AGUILERA-SALGADO*, Alexander CÁRDENAS-MEJÍA**
}

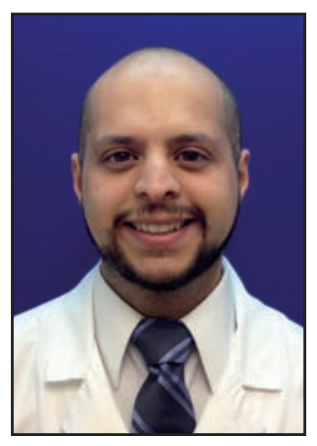

Aguilera Salgado, A.
Resumen

Introducción y Objetivo. El síndrome de Möbius es un trastorno congénito que afecta al nervio facial y al abducens, provocando parálisis facial uni o bilateral y estrabismo. Su tratamiento consiste en lograr la reanimación facial con la transferencia de un músculo funcional libre. Típicamente, se ha realizado en dos o más etapas, siendo controvertido el tiempo entre las cirugías, así como el nervio donador ideal para el colgajo (rama maseterina del trigémino o espinal accesorio).

Describimos nuestra cirugía de reanimación facial en Síndrome de Möbius completo mediante transferencia en un solo tiempo de gracilis libre bilateral, usando como nervio donador la rama maseterina del trigémino o el nervio espinal accesorio. Evaluamos resultados y determinamos si es un procedimiento efectivo y seguro.

Material y Método. Entre marzo de 2014 y octubre de 2016, sometimos a transferencia en un solo tiempo de gracilis libre bilateral a 4 pacientes con Síndrome de Möbius. Realizamos exploración física y neurológica detallada para determinar los nervios donadores. Tradicionalmente nuestra primera elección es el trigémino, sin embargo estaba afectado en 2 pacientes, por lo que en ellos utilizamos el nervio espinal accesorio.

El seguimiento promedio fue de 17 meses Hicimos la evaluación postoperatoria utilizando la escala de valoración estética y funcional de Terzis, la escala de excursión de la sonrisa de Chuang, y una escala de satisfacción postoperatoria.

Resultados. De los 4 pacientes operados 2 eran varones y 2 mujeres. Realizamos 8 transferencias musculares libres en 4 tiempos quirúrgicos. Utilizamos los vasos faciales como receptores en todos los casos. Empleamos el nervio trigémino como donador en 2 pacientes y en los otros 2 el nervio espinal accesorio. No hubo complicaciones ni pérdidas de colgajos. Todos los pacientes lograron un mínimo de III en la escala de valoración estética y funcional de Terzis, y de IV en los que usamos el espinal accesorio como donador. El resultado mínimo utilizando la escala de excursión de la sonrisa de Chuang fue de 2, pero mejorando a 3 en los que usamos el espinal accesorio. El promedio en la escala de satisfacción postoperatoria fue de 3 , pero en el paciente con mayor tiempo de seguimiento fue de 4 .

Conclusiones. La cirugía de reanimación facial en pacientes con síndrome de Möbius completo mediante transferencia en un solo tiempo quirúrgico de gracilis libre bilateral es un procedimiento seguro y efectivo. La selección cuidadosa de los pacientes, una exploración física completa cuidadosa, la selección de nervios motores donadores, la realización impecable de la cirugía y un programa de rehabilitación postoperatoria correcto, son indispensables para tener éxito.

\begin{tabular}{|c|c|c|}
\hline Palabras clave & \multicolumn{2}{|c|}{$\begin{array}{l}\text { Síndrome Möbius, Reanimación facial, } \\
\text { Transferencia libre muscular, } \\
\text { Gracilis, Parálisis facial. }\end{array}$} \\
\hline \multicolumn{2}{|c|}{ Nivel de evidencia científica } & 4c Terapéutico \\
\hline \multirow{2}{*}{\multicolumn{2}{|c|}{$\begin{array}{l}\text { Recibido [esta versión] } \\
\text { Aceptado }\end{array}$}} & 20 febrero/2018 \\
\hline & & 14 marzo/2018 \\
\hline
\end{tabular}

\begin{abstract}
Background and Objective. Möbius syndrome is a congenital disorder that affects facial and abducens cranial nerves, resulting in uni or bilateral facial palsy and strabismus. The treatment is facial reanimation with free functional muscle transfer. Typically, facial reanimation has been realized in 2 or more stages with controversial issues, such as the amount of time between the stages and the ideal donor nerve (masseter or spinal accessory).

We describe our one-stage facial reanimation surgery for complete Möbius Syndrome using 2 free gracilis muscle flaps, and bilateral trigeminal or spinal nerves as a donor. We evaluate our outcomes and determine if this is an effective and safe procedure.

Methods. Between March 2014 and October 2016, 4 patients with complete Möbius Syndrome were operated using bilateral gracilis free muscle transfers in one surgical time. Detailed physical and neurologic examinations were performed with special emphasis on which cranial nerves were involved. Traditionally our first choice of nerve donor has been the trigeminal nerve, but it was involved in 2 patients, so in theme we used the spinal nerve as a donor to innervate 2 free gracilis flaps for smile reconstruction.

The mean postoperative follow-up time was 17 months. Outcomes were assessed using the Terzis functional and aesthetic grading system, Chuang's smile excursion score, and a questionnaire to evaluate patient satisfaction.

Results. A total of 4 patients were included: 2 male and 2 female. We performed 8 free gracilis muscle transfers in 4 surgical times. We used the facial vessels in every flap, and the trigeminal nerve as a donor in 2 patients and in the 2 remaining, the spinal nerve. We have no complications nor flap failures. All patients achieved at least score of III using the Terzis functional and aesthetic grading system, and IV in those with spinal nerve as a donor. Likewise, the minimum score using Chuang's smile excursion score was 2, but in those with spinal nerve as a donor we got a score of 3. Finally the mean satisfaction score was 3, but the patient with the longest follow-up time gave a 4 .
\end{abstract}

Conclusions. One-stage facial reanimation surgery for complete Möbius Syndrome using 2 free gracilis muscle flaps is an effective and safe procedure. Careful patient selection, adequate physical evaluation, motor nerve donor selection, flawless surgery performance, and adequate postoperative rehabilitation program, are mandatory in order to obtain success.

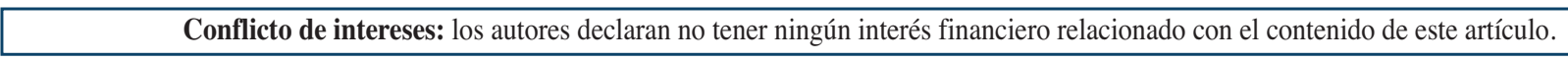

Video complementario al artículo en: www.ciplaslatin.com

\begin{tabular}{|c|c|c|}
\hline Key words & \multicolumn{2}{|c|}{$\begin{array}{l}\text { Möbius Syndrome, Facial reanimation } \\
\text { Free functional muscle transfer, } \\
\text { Gracilis, Facial palsy. }\end{array}$} \\
\hline & & $4 \mathrm{c}$ Therapeutic \\
\hline cen & & 20 february/2 \\
\hline
\end{tabular}

\begin{tabular}{c}
\hline $\begin{array}{c}\text { Video complementario al artículo en: } \\
\text { www.ciplaslatin.com }\end{array}$ \\
\hline
\end{tabular}

* Médico Adscrito al Servicio de Cirugía Plástica y Reconstructiva del Instituto Nacional de Pediatría, Ciudad de México, México.

** Cirujano Plástico, Jefe de la Clínica de Parálisis Facial y Nervio Periférico de la División de Cirugía Plástica y Reconstructiva, Hospital General Dr. Manuel Gea González, Ciudad de México, México. 


\section{Introducción}

Las primeras descripciones de la asociación entre la parálisis congénita del nervio facial y del abducens o motor ocular externo fueron realizadas por Von Graefe en 1880, seguido por Harlam en 1881 y Christholm en 1882. Sin embargo, no fue hasta el año de 1892 cuando el neurólogo alemán Paul Julius Möbius presentó una serie de 43 pacientes en los que identificó la asociación de la parálisis facial congénita con otras malformaciones, elaborando la clásica descripción del síndrome que después recibiría su nombre: parálisis facial no progresiva simétrica o asimétrica y ausencia de la abducción de los dos ojos, aunada con alteraciones de otros nervios craneales, principalmente de los pares craneales V, IX y XII..$^{(1)}$

El Síndrome de Möbius es un trastorno congénito raro, por lo cual no es fácil encontrar datos estadísticos respecto a su incidencia. Se calcula 1 caso por cada 50.000 nacidos

Tabla I. Espectro clínico del síndrome de Möbius.

\begin{tabular}{|c|c|}
\hline Área & Alteraciones \\
\hline Orofaríngeas & $\begin{array}{c}\text { Hipoplasia y asimetría en el tamaño de la } \\
\text { lengua } \\
\text { Cambios en la úvula } \\
\text { Regurgitación } \\
\text { Incompetencia laríngea } \\
\text { Parálisis de cuerdas vocales }\end{array}$ \\
\hline Craneofaciales & $\begin{array}{c}\text { Telecanto } \\
\text { Aplanamiento del puente nasal } \\
\text { Micrognatia } \\
\text { Paladar alto o hendido } \\
\text { Defectos del pabellón auricular } \\
\text { Hipoacusia por estenosis severa del CAI* }\end{array}$ \\
\hline Musculoesqueléticas & $\begin{array}{l}\text { Sindactilia, braquidactilia, clinodacitilia, } \\
\text { ectrodactilia, campilodactilia } \\
\text { Pie plano o equinovaro } \\
\text { Fémur valgo } \\
\text { Ausencia de músculo pectoral } \\
\text { (Síndrome de Poland) }\end{array}$ \\
\hline $\begin{array}{l}\text { Neurológicas y } \\
\text { Neurosensoriales }\end{array}$ & $\begin{array}{c}\text { Calcificaciones cerebrales (tallo cerebral) } \\
\text { Quistes aracnoideos } \\
\text { Atrofia cerebral } \\
\text { Alteración de Dandy-Walker } \\
\text { Hidrocefalia } \\
\text { Convulsiones } \\
\text { Hipoestesias } \\
\text { Hipotonía } \\
\text { Disfunción respiratoria primaria }\end{array}$ \\
\hline Trastornos mentales & $\begin{array}{c}\text { Retraso psicomotor } \\
\text { Trastornos de coordinación motora } \\
\text { Retardo mental } \\
\text { Autismo }\end{array}$ \\
\hline Oftalmológicos & $\begin{array}{c}\text { Defectos refactivos y ambliopía } \\
\text { Alteraciones palpebrales (hipoestesia, } \\
\text { ptosis, epicanto) } \\
\text { Lagrimeo } \\
\text { Queratocono } \\
\text { Estrabismo: endotropia con limitación } \\
\text { a la abducción }\end{array}$ \\
\hline
\end{tabular}

vivos. Aproximadamente sólo 300 casos están recogidos en la literatura en inglés hasta el año 2006, mientras que en México existen 3 publicaciones recientes del Síndrome de Möbius presentadas como reporte de casos en los Estados de México, Tamaulipas y Tabasco. ${ }^{(2-4)}$

El Síndrome de Möbius comprende un espectro amplio de manifestaciones clínicas que nos permiten hacer el diagnóstico (Tabla I). Además de la afectación del VI y VII pares craneales podemos también encontrar afectación de otros pares craneales como son el II, V, IX, X, XI y XII, en mayor o menor medida. ${ }^{(1)}$

Dada la heterogenicidad y la variabilidad de la presentación clínica del síndrome, Terzis y col. propusieron una clasificación del mismo que permite a los cirujanos reconstructivos identificar las deficiencias en los nervios motores, $\mathrm{y}$ en base a esto establecer una estrategia reconstructiva específica para cada paciente:

- Möbius completo: parálisis bilateral del VI y VII nervios craneales.

- Möbius incompleto: características clínicas del síndrome pero con función motora residual en un lado de la cara.

- Möbius-like: parálisis facial unilateral pero con otros nervios craneales afectados.

En esta publicación de Terzis, el VI par estaba afectado en el $81 \%$ de los casos, mientras que el XII y el V pares craneales lo estaban en el 55 y el 23\% de los casos respectivamente. De todos los nervios motores potencialmente donadores, el XI fue el menos afectado, con un $9 \%$ de los casos. La mayoría de los pacientes presentaron afectación de 2 o más nervios craneales. ${ }^{(1)}$ Por otra parte, un estudio realizado en 2014 en la División de Cirugía Plástica del Hospital General Dr. Manuel Gea González de la Ciudad de México (México), incluyó el análisis de 115 pacientes con diagnóstico de Síndrome de Möbius en sus 3 presentaciones los cuales fueron sometidos a evaluación clínica completa por un equipo multidisciplinario, siendo el $45 \%$ varones y el 55\% mujeres, con afectación del VI par craneal en el $100 \%$ de los casos, estrabismo en el $62.6 \%$, pie equino varo en el $46.1 \%$, sindactilia simple en el $15.7 \%$, paladar hendido en el $17.4 \%$, y micrognatia y síndrome de Poland en el 9.6\%, entre otros. Hasta este momento, este estudio incluye la serie más grande de pacientes con Síndrome de Möbius en un solo centro hospitalario. ${ }^{(5)}$

\section{Protocolos internacionales de tratamiento}

Dentro del espectro de tratamiento, el pilar fundamental lo constituyen los procedimientos quirúrgicos que están dirigidos principalmente a corregir alteraciones oculares, de las extremidades y de la cara inexpresiva característica. $^{(6)}$

Hablando de la reanimación dinámica de la cara, anteriormente se utilizaban distintos colgajos musculares locales como el masetero, el temporal y el platisma. Sin embargo, con el advenimiento de las técnicas microqui- 
rúrgicas, la trasferencia muscular libre se ha convertido en la técnica de elección,(7) siendo el músculo gracilis la primera opción debido a su fácil acceso, tamaño, forma, longitud, vascularización, nervio motor único largo, adecuada fuerza muscular y excursión, y morbilidad mínima del sitio donador.

Una parte fundamental en la toma de decisiones para realizar la transferencia muscular es una adecuada evaluación y exploración física preoperatoria, complementada con estudios electrofisiológicos que permitan identificar los nervios afectados y los posibles donadores. Dicha evaluación permite al cirujano reconstructivo desarrollar una secuencia óptima reconstructiva específica para cada paciente en particular. Toda esta exploración debe ser documentada en fotografías y almacenada en videos para establecer una evaluación objetiva pre y postoperatoria.

En cuanto a los nervios craneales que pueden funcionar como nervios donadores para el colgajo libre están el nervio trigémino, el espinal y el hipogloso. En caso de que el paciente presente función residual del nervio facial contralateral, es decir que se trate de un caso de Möbius incompleto, es preferible neurotizar el colgajo de gracilis con un injerto nervioso cruzado de nervio facial contralateral.

Bianchi describe la transferencia de músculo gracilis en 12 pacientes en los que utilizó como nervio motor donador el del músculo masetero, recogiendo un $84.4 \%$ de satisfacción con los resultados estéticos y funcionales, y logrando una simetría facial en reposo de buena a excelente en todos los pacientes. ${ }^{(8)}$ Terzis en 2003 presenta un grupo de 25 pacientes sometidos a reanimación facial con colgajo libre de gracilis, en los cuales utilizó como donador el nervio facial contralateral en 10 casos, en 5 el nervio espinal, en 4 la rama maseterina, en 4 más el nervio hipogloso y en los últimos 2 empleó nervios intraplexo braquial. En todos los casos el resultado fue bueno, logrando contracción cercana a la normal y altos índices de satisfacción por parte de sus pacientes. ${ }^{(9)}$

Finalmente, tanto Zucker, ${ }^{(10)}$ como Chuang ${ }^{(11)}$ y Cárdenas presentan como técnica de primera elección la reanimación facial con músculo gracilis utilizando en todos los casos, cuando es posible, el nervio maseterino como nervio donador debido a la baja morbilidad que representa para el paciente, la posibilidad de evitar injertos nerviosos de interposición, así como su potencial para una buena adaptación cerebral.

En cuanto al número de tiempos quirúrgicos, la reconstrucción clásica se ha realizado en 2 tiempos, reconstruyendo primero un lado de la cara y tiempo después el lado contralateral, pero teniendo como punto principal de controversia el tiempo que debe transcurrir entre una cirugía y otra y la selección de los nervios que van a neurotizar el colgajo libre. ${ }^{(12)}$ Terzis y Noah aportan la reanimación bilateral en 6 pacientes con Síndrome de Möbius realizada en 4 tiempos quirúrgicos y utilizando injertos para elongar los nervios donadores, entre ellos el nervio espinal, la rama maseterina, el nervio hi- pogloso y el C7 ipsilateral, con un periodo de entre 7 meses a 2 años entre la colocación del injerto y la transferencia muscular libre. ${ }^{(9)}$ Zuker realizó la transferencia muscular libre en 10 pacientes utilizando el nervio maseterino como donador y dejando un lapso de 3 meses entre un lado de la cara y el otro. ${ }^{(13)}$ Lischez, por su parte, recoge 2 casos en los que dejó un periodo de 1 año entre una cirugía y otra. ${ }^{(14)}$

Los únicos casos en la literatura en los que se presenta la restauración dinámica de la sonrisa en pacientes con Síndrome de Möbius completo en un solo tiempo quirúrgico son los de Woollard, ${ }^{(8)}$ quien utilizó la rama maseterina como nervio donador para inervar colgajos de dorsal ancho segmentarios bilaterales en 20 pacientes; y recientemente Chuang publicó su experiencia en 6 pacientes en los que utilizó como nervio donador el nervio espinal para inervar el músculo gracilis transferido de forma bilateral en un solo tiempo quirúrgico, con inicio de movimientos a los 3 meses hasta lograr una sonrisa independiente a los 6 meses en niños, $y$ al año en adultos..$^{(15)}$

En el presente artículo nos proponemos describir nuestra cirugía de reanimación facial en pacientes con Síndrome de Möbius completo mediante transferencia en un solo tiempo quirúrgico de gracilis libre bilateral, usando como nervio donador la rama maseterina del trigémino o el nervio espinal accesorio. También, evaluar los resultados obtenidos mediante el uso de escalas internacionales validadas y determinar si se trata un procedimiento efectivo y seguro.

\section{Material y método}

Realizamos el estudio en el Servicio de Cirugía Plástica y Reconstructiva del Hospital General Dr. Manuel Gea González, en la Ciudad de México (México). Incluimos 4 pacientes con diagnóstico de Síndrome de Möbius completo, confirmado por exploración y estudio neurofisiológico, que no habían sido sometidos a ninguna cirugía facial previa ni a algún otro tipo de procedimiento de reanimación facial. Todos los pacientes contaban con expediente clínico fotográfico completo, con videos pre y postoperatorios.

Una vez seleccionados los pacientes, fueron sometidos a cirugía de reanimación facial mediante transferencia de colgajo libre de gracilis bilateral en un solo tiempo quirúrgico y mantenidos en vigilancia estrecha durante 5 días en el hospital, monitorizando los colgajos libres; posteriormente, fueron seguidos en la consulta externa, documentando siempre los resultados con fotografías y videos. Una vez transcurrido un periodo mínimo de 12 meses a partir de la cirugía, realizamos la evaluación de los resultados utilizando las siguientes escalas:

- Escala de valoración estética y funcional de Terzis (Tabla II).

- Escala de excursión de la sonrisa de Chuang (Tabla III).

- Escala de satisfacción de los pacientes con los resultados (Tabla IV). 
Tabla II. Escala de valoración estética y funcional de Terzis.

\begin{tabular}{|c|c|c|}
\hline Grupo & Resultado & \multicolumn{1}{c|}{ Descripción } \\
\hline I & Malo & Deformidad, no hay contracción \\
\hline II & Pobre & Contracción minima, voluminoso \\
\hline III & Moderado & Movimiento en masa, contracción moderada \\
\hline IV & Bueno & Simetria, contracción completa \\
\hline V & Excelente & Sonrisa completa, muestra dientes \\
\hline
\end{tabular}

Tabla III. Escala de excursión de la sonrisa de Chuang.

\begin{tabular}{|c|c|c|c|}
\hline Clasificación & Dientes Visibles & Contractura & Sincinesia \\
\hline 0 & Ninguno & -0.5 & -0.5 \\
\hline 1 & Incisivo Central & -0.5 & -0.5 \\
\hline 2 & Incisivo Lateral & -0.5 & -0.5 \\
\hline 3 & Canino & -0.5 & -0.5 \\
\hline 4 & Premolar o más & -0.5 & -0.5 \\
\hline
\end{tabular}

\begin{tabular}{|c|c|}
\hline CALIFICACIÓN & DESCRIPCIÓN \\
\hline 1 & Se arrepiente de la cirugía \\
\hline 2 & $\begin{array}{l}\text { No es aceptable, pero no se arrepiente de la } \\
\text { cirugía }\end{array}$ \\
\hline 3 & $\begin{array}{c}\text { Aceptable, pero no es la sonrisa que esperaba, } \\
\text { necesita mejorias }\end{array}$ \\
\hline 4 & Satisfecho, necesita pequeños ajustes \\
\hline 5 & Completamente satisfecho \\
\hline
\end{tabular}

Tabla V. Grupo de estudio.

\begin{tabular}{|c|c|c|c|}
\hline Sexo & Edad & Nervio Empleado & $\begin{array}{c}\text { Seguimiento } \\
\text { (Meses) }\end{array}$ \\
\hline Masculino & 4 & Espinal & 25 \\
\hline Femenino & 22 & Maseterina & 12 \\
\hline Masculino & 6 & Espinal & 20 \\
\hline Femenino & 5 & Maseterina & 12 \\
\hline
\end{tabular}

\section{RESULTADOS}

Del total de 4 pacientes con diagnóstico de Síndrome de Möbius completo, 2 fueron varones y 2 mujeres, con un promedio de edad de 9 años (rango de 4 a 22 años). A todos se les realizó una historia clínica y exploración física completas, identificando la integridad tanto del nervio trigémino como del nervio espinal accesorio mediante pruebas clínicas y electromiografía.

Todos los pacientes fueron sometidos a reanimación facial en un solo tiempo quirúrgico con colgajos libres de gracilis bilaterales, realizando un total de 8 colgajos libres de gracilis de los cuales 4 fueron neurotizados con la rama maseterina del trigémino, y los 4 restantes con el nervio espinal accesorio (Tabla V). Todas las intervenciones fueron realizadas por los mismos cirujanos (Aguilera/Cárdenas).

\section{Técnica quirúrgica}

En la intervención trabajaron 2 equipos quirúrgicos de forma simultánea. El primer equipo inició el abordaje facial derecho mediante una incisión preauricular con una extensión temporal y otra hacia el reborde mandibular, disecando con electrocauterio en plano sub-SMAS, por arriba de la fascia parotídea. Continuamos hasta identificar el borde medial de la parótida y del músculo masetero, iniciando la disección con tijera de Stevens y pinza bipolar de electrocauterio hasta identificar la vena facial, la cual referimos y disecamos en dirección a la comisura bucal, identificando en el trayecto la bolsa de Bichat que resecamos completamente, teniendo cuidado siempre de llevar a cabo una correcta identificación y preservación del conducto de Stenon. Una vez concluido este paso, volvemos al punto anterior para identificar la arteria facial, que referimos y disecamos de igual forma hacia la comisura bucal. Colocamos 2 clips quirúrgicos en el extremo más distal de los vasos y los redirigimos en sentido proximal.

Procedemos entonces a identificar el músculo orbicular de los labios, el modiolo, y los músculos cigomáticos, colocando un punto de monocryl 3-0 en cada uno de estos sitios, y verificando que en el momento de traccionarlos se logra recrear el surco nasogeniano que se aprecia normalmente en una sonrisa. Con estas referencias tomamos las medidas del músculo que vamos a colocar en esta zona, midiendo la anchura y la longitud tanto del borde superior como del borde inferior.

En los casos en los que realizamos la neurotización con la rama maseterina, identificamos el borde inferior del arco cigomático y, mediante palpación, lo seguimos hasta la escotadura medial del cuerpo del arco donde realizamos una incisión transversal de $2 \mathrm{~cm}$ sobre el SMAS con electrocauterio, continuando con disección roma con tijera y pinza vascular en el espesor del músculo hasta identificar la rama maseterina, la cual estimulamos para verificar la adecuada contracción muscular del masetero, cortándola posteriormente en el extremo distal, redirigiéndola en sentido proximal y colocando gelfoam en el área de la disección.

En los casos en los que utilizamos el nervio espinal accesorio como nervio donador, realizamos una incisión longitudinal en el borde posterior del músculo esternocleidomastoideo ipsilateral, continuando la disección con tijera de Stevens hasta encontrar el nervio espinal accesorio en la superficie posterior del músculo trapecio en su cara lateral, el cual confirmamos con neuroestimulador (Fig. 1). Continuamos la disección tan distal como sea posible, hasta su división en 2 o 3 ramas. En esta región 


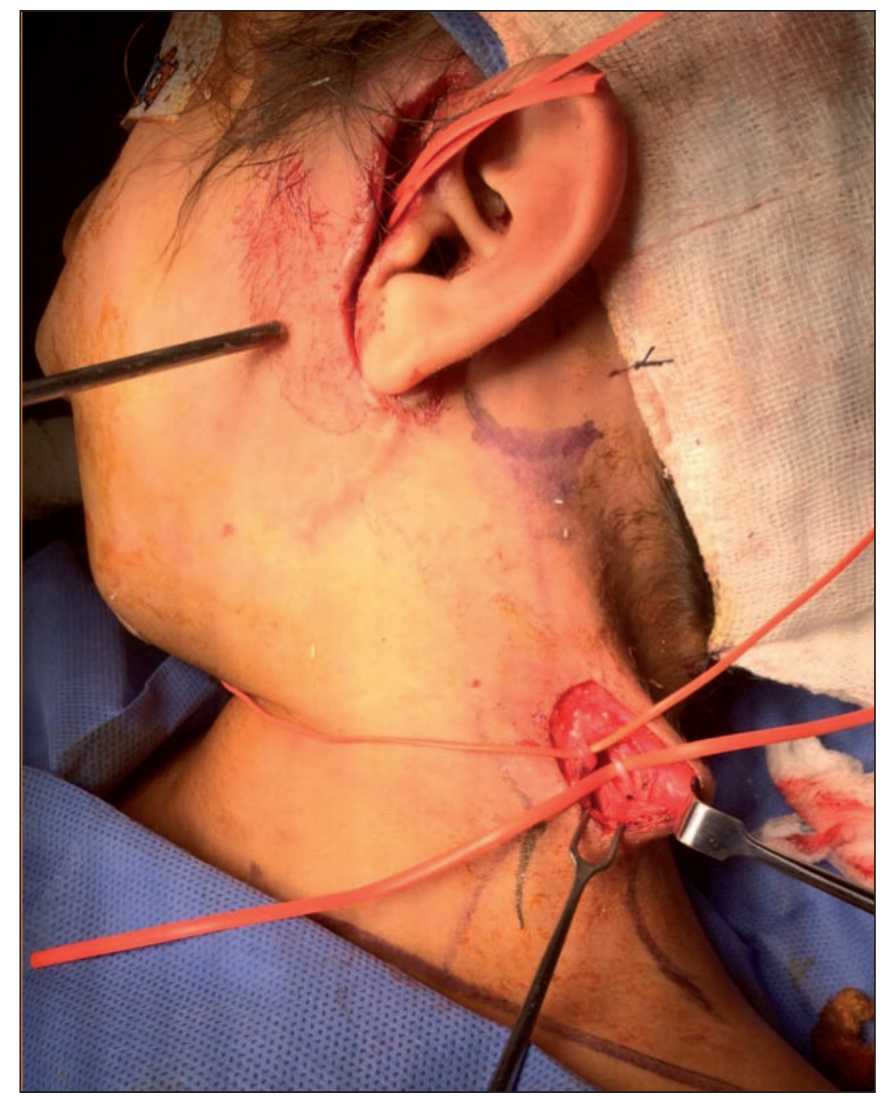

Fig. 1. Disección del nervio espinal accesorio hacia la superficie posterior del músculo trapecio.

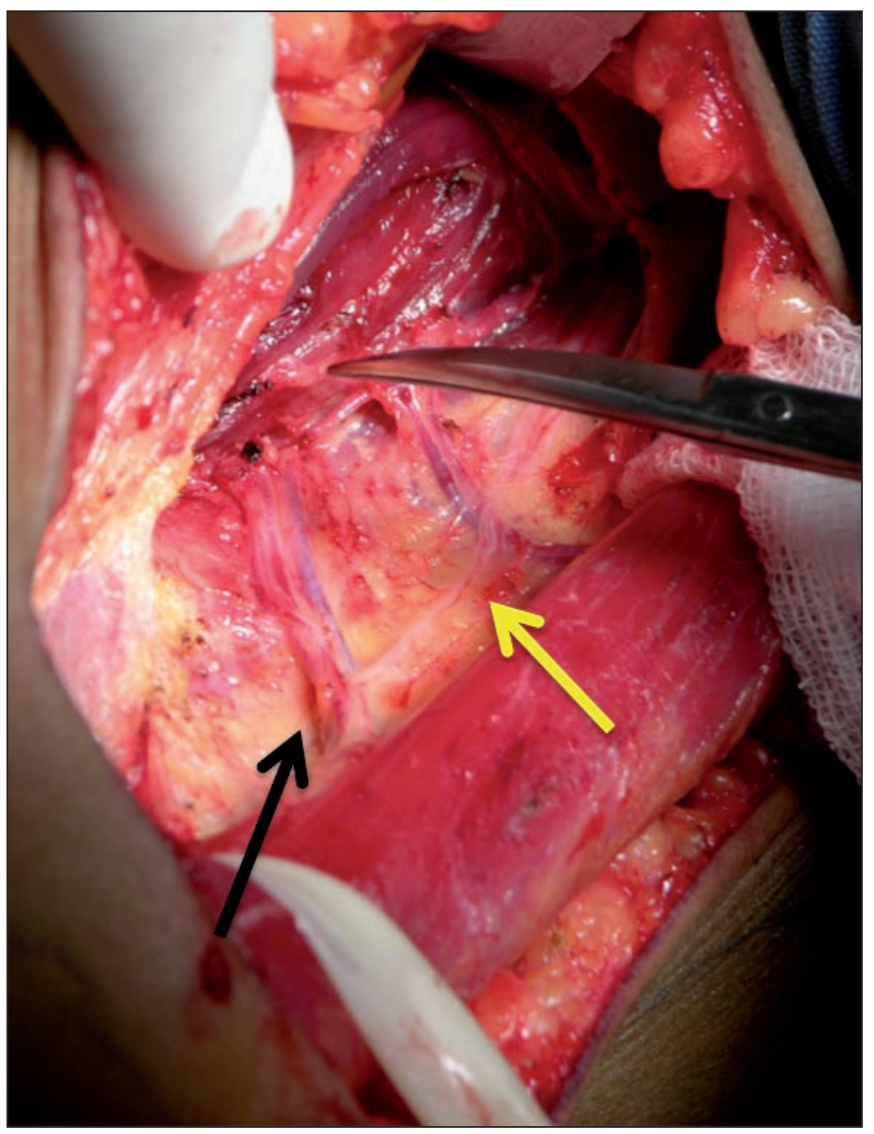

Fig. 2. Disección del pedículo vascular (flecha negra) y del nervio obturador (flecha amarilla).

seccionamos el nervio y lo llevamos hacia el ángulo mandibular a través de un túnel creado por debajo del platisma, dando por terminada la disección de la cara.

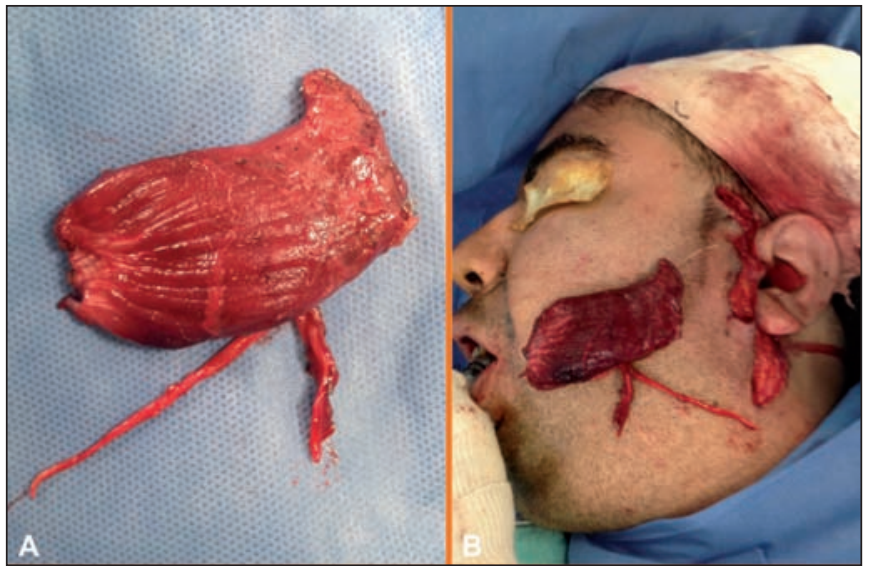

Fig. 3. A. Disección finalizada del músculo gracilis, listos para iniciar tiempo microquirúrgico. B. Colocado en la superficie de la mejilla representando su posición final.

Simultáneamente, el segundo equipo quirúrgico inicia la disección del colgajo de gracilis derecho con una incisión longitudinal siguiendo el borde inferior del músculo aductor largo en la cara interna del muslo, de $7 \mathrm{~cm}$ de longitud aproximadamente, iniciando a $3 \mathrm{~cm}$ del pubis cerca del pliegue inguinal, y profundizando por planos con electrocauterio hasta identificar las fibras del músculo gracilis. Con disección digital rodeamos el músculo en su extremo tanto proximal como distal y colocamos un drenaje de Penrose en cada extremo que nos servirá como tracción del músculo.

Identificamos el nervio obturador en el tercio proximal del músculo, verificando la contracción adecuada del gracilis mediante neuroestimulador, y siguiendo dicho nervio hasta su origen en el agujero obturador. A continuación, disecamos la región inferior del aductor largo en sentido medial hasta encontrar el pedículo vascular, el cual disecamos completamente hasta llegar a los vasos femorales y realizando hemostasia cuidadosa de todas las ramas vasculares que encontramos, tratando de disecar de manera independiente la arteria de ambas venas del pedículo (Fig. 2).

Tomando como referencia las medidas realizadas por el primer equipo, realizamos el corte del músculo con 2 disparos de engrapadora lineal, uno en sentido longitudinal dirigiendo la mayor longitud hacia el tendón del músculo gracilis, y otro en sentido transversal, finalizando el corte con electrocauterio. Una vez completada la disección de la cara. realizamos el corte del músculo, colocamos grapas vasculares en cada uno de los vasos del pedículo, seccionamos el nervio obturador, y entonces estamos listos para iniciar el primer tiempo de la microcirugía (Fig. 3).

Realizamos un corte longitudinal en el extremo del músculo donde se encuentra el tendón, formando una $\mathrm{V}$ con el mismo. Tomamos nuestras riendas de referencia de monocryl y colocamos un punto en cada extremo de la $\mathrm{V}$ y otro en la parte central de la misma, asegurándonos de que sea éste el punto de monocryl que está tomando el modiolo. Bajamos nuestros puntos y ajustamos el otro 


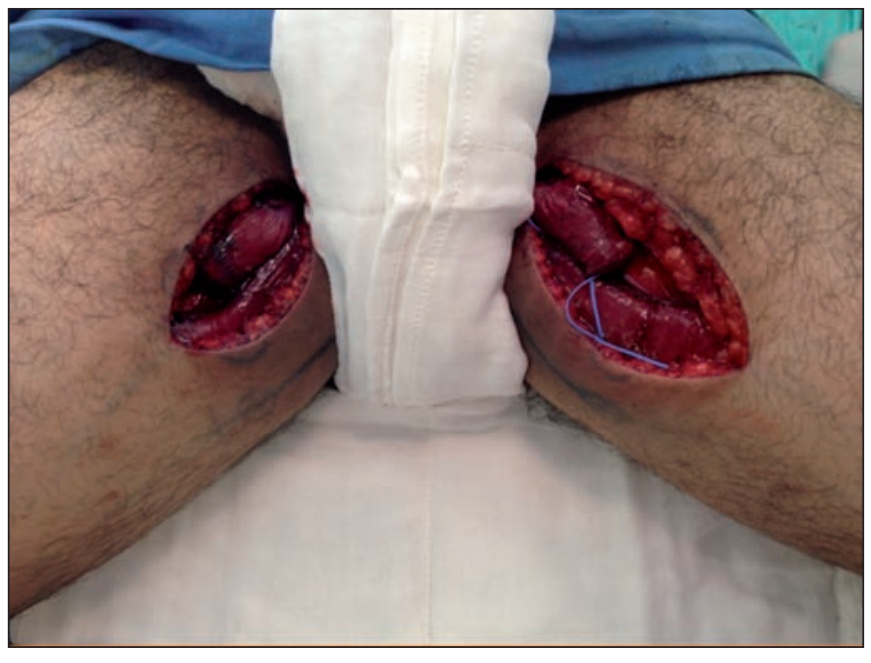

Fig. 4. Disección final bilateral en el mismo tiempo quirúrgico.

extremo del músculo con 2 puntos de monocryl hacia la fascia temporal. Una vez fijo el músculo, iniciamos la coaptación nerviosa del nervio obturador a la rama maseterina, o en su caso al nervio espinal, mediante 2 puntos simples con nylon 10-0 y colocando gel de fibrina una vez alineados los extremos. Procedemos entonces con las anastomosis vasculares hacia los vasos faciales, realizándolas con puntos simples de nylon 10-0. Verificamos la permeabilidad de nuestras anastomosis y procedemos al cierre por planos de las heridas.

Una vez concluido el tiempo de microcirugía, continuamos con el lado contralateral realizando los mismos pasos y en el mismo orden.

El promedio de la duración total del procedimiento fue de 6.5 horas (Fig. 4).

\section{Postoperatorio y seguimiento}

Los pacientes permanecieron hospitalizados durante 5 días, siendo vigilados de forma horaria con doppler para verificar la permeabilidad vascular de los músculos gracilis. No se presentaron complicaciones en ningún caso y ningún colgajo se perdió. De igual forma, no fue necesario reexplorar quirúrgicamente a ningún paciente.

Una vez egresados del hospital, llevamos a cabo seguimiento en consulta externa donde se les pidió a los pacientes que trabajaran en ejercicios para estimular los músculos cuyos nervios habíamos empleado como donadores: masticación y elevación de hombros, para así favorecer la reinervación del gracilis. Notamos diferencia en cuanto al tiempo en el cual la contracción muscular comenzó a ser evidente para ambos lados de la cara, que fue de 12 semanas como promedio para el lado derecho (mínimo 10 semanas, máximo 24 semanas) y de 15 semanas para el lado izquierdo (mínimo 12 semanas, máximo 30 semanas). Una vez que aparecía la contracción, iniciamos rehabilitación de la sonrisa entrenando al paciente para identificar el movimiento que desencadena su sonrisa, y recrearlo frente a un espejo para favorecer la adaptación cortical.
Tabla VI. Resultados al emplear la escala de valoración estética y funcional de Terzis.

\begin{tabular}{|c|c|c|}
\hline Nervio Donador & Valor Preoperatorio & Valor Posoperatorio \\
\hline Espinal & I & V \\
\hline Espinal & I & IV \\
\hline Maseterino & I & III \\
Maseterino & I & III \\
\hline Promedio & I & 3.5 \\
\hline
\end{tabular}

Tabla VII. Resultados al emplear la escala de excursión de la sonrisa de Chuang.

\begin{tabular}{|c|c|c|}
\hline Nervio Donador & Valor Preoperatorio & Valor Posoperatorio \\
\hline Espinal & 0 & 3 \\
\hline Espinal & 0 & 3 \\
\hline Maseterino & 1 & 2 \\
\hline Maseterino & 0 & 2 \\
\hline Promedio & 0.25 & 2.5 \\
\hline
\end{tabular}

El seguimiento promedio hasta el momento ha sido de 17 meses, con un mínimo de 12 meses y un máximo de 25 meses, tiempo en el que a todos los pacientes se les han tomado fotografías y videos mientras realizan una sonrisa en máxima excursión.

\section{Resultados funcionales y satisfacción}

Comparamos las fotografías y los videos postoperatorios con los preoperatorios y 2 cirujanos plásticos independientes, que no formaban parte del equipo quirúrgico, valoraron los resultados utilizando la escala de valoración estética y funcional de Terzis (Tabla II). Encontramos valores iniciales de I en todos los casos, logrando valores postoperatorios de mínimo III en todos los casos, y logrando IV en los pacientes en los que utilizamos el nervio espinal como donador, con un promedio de 3.5 para el total (Tabla VI).

De igual forma, utilizamos la escala de excursión de la sonrisa de Chuang que evalúa el resultado dependiendo de la visibilidad de los dientes durante una sonrisa completa e incluye 5 categorías (Tabla III). Todos nuestros pacientes alcanzaron por lo menos el 2 de calificación, mejorando de una calificación inicial de promedio de 0.25 a una calificación postoperatoria de 2.5 de promedio; logramos hasta el momento las mejores calificaciones en los 2 pacientes en los que utilizamos como nervio donador el espinal accesorio ( 3 de calificación frente a 2 de calificación en los pacientes en los que usamos la rama maseterina). Ningún paciente presentó sincinesias ni contracturas (Tabla VII).

Finalmente, aplicamos un cuestionario para evaluar el grado de satisfacción de los pacientes con los resultados 
Tabla VIII. Resultados al emplear la escala de satisfacción de los pacientes con los resultados.

\begin{tabular}{|c|c|c|}
\hline Nervio Donador & Valor Preoperatorio & Valor Posoperatorio \\
\hline Espinal & NO APLICA & 4 \\
Espinal & NO APLICA & 3 \\
\hline Maseterino & NO APLICA & 3 \\
\hline Maseterino & NO APLICA & 2 \\
\hline Promedio & NO APLICA & 3 \\
\hline
\end{tabular}

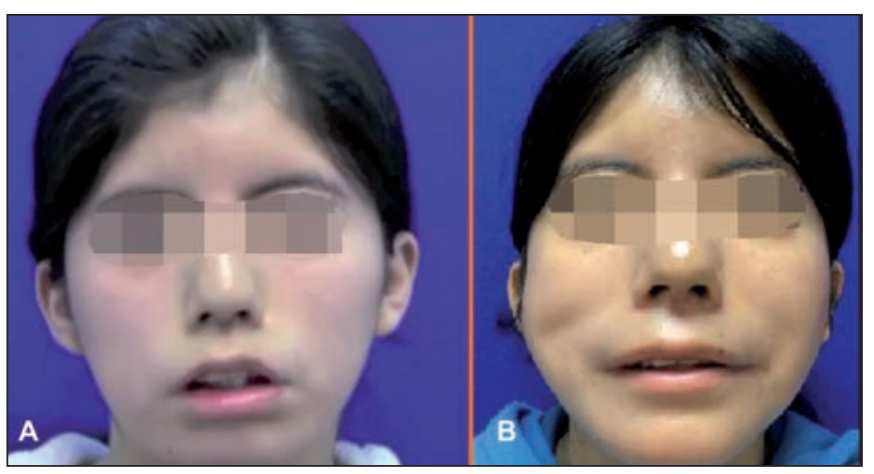

Fig. 5. A. Preoperatorio de paciente cuyos colgajos fueron neurotizados con rama maseterina. B. Postoperatorio a los 12 meses.

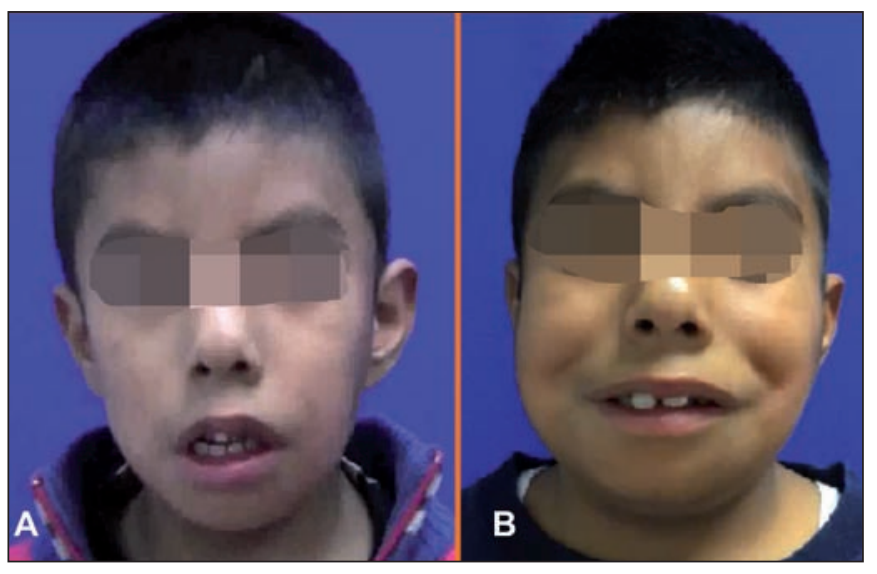

Fig. 6. A. Preoperatorio de paciente cuyos colgajos fueron neurotizados con nervio espinal. B. Postoperatorio a los 20 meses.

postoperatorios, asignando un valor del 1 al 5 dependiendo lo satisfechos que se encontraran con los resultados, si creen que precisan más ajustes en su sonrisa y si volverían a someterse al procedimiento (Tabla IV). Completamos el cuestionario con un evaluador independiente. Los resultados hasta el momento revelan un promedio de grado de satisfacción de 3 , siendo el primer caso operado y por ende el de mayor tiempo de seguimiento el que obtuvo 4 de calificación en comparación con el 2 otorgado por el de menor periodo de seguimiento (Tabla VIII). Pensamos que a medida que avancemos con la rehabilitación de la sonrisa y con el tiempo de seguimiento, el grado de satisfacción de los pacientes será mayor (Fig. 5 y 6).

Ningún paciente presentó dolor asociado en cuello u hombros, ni dificultad con la masticación. Los 4 pacientes deberán completar un periodo de rehabilitación de la sonrisa de 30 meses ( 2 años y medio), ya que se trata de una media recomendable por ser este el tiempo hasta el cual el paciente es capaz de seguir mejorando para incrementar el grado de excursión de la misma, además de tratar de lograr una mejor adaptación cortical, por lo que estos valores deberán de mejorar en proporción directa cuanto mayor sea el tiempo de seguimiento que les demos.

\section{Discusión}

Los procedimientos de reanimación dinámica facial para pacientes con Síndrome de Möbius están descritos en la literatura y van desde la trasferencia de músculos regionales de la cara: masetero, temporal y platisma, hasta la transferencia muscular libre, principalmente del músculo gracilis, la cual se ha convertido en la técnica de elección en estos pacientes desde el advenimiento de las técnicas microquirúrgicas. ${ }^{(7)}$ El objetivo en estos casos siempre es lograr una sonrisa simétrica, espontánea, con adecuada excursión del músculo trasferido y estéticamente acorde a la cara del paciente.

Los pacientes con Síndrome de Möbius clásico tienen afectados ambos lados de la cara, dando la apariencia de máscara sin expresión facial. ${ }^{(16)}$ Para su reanimación facial están descritas distintas técnicas y protocolos quirúrgicos debido a que por la naturaleza misma de la enfermedad, son pacientes que pueden llegar a requerir múltiples cirugías a lo largo de su vida.

Terzis y $\operatorname{Noah}^{(9)}$ describieron en 2003 su técnica de reanimación facial en un grupo de pacientes, la cual consistía en 4 tiempos quirúrgicos y dejando un periodo de entre 7 meses a 2 años para realizar la transferencia muscular libre. Tanto Zucker como Cárdenas presentan como técnica de primera elección la reanimación facial con músculo gracilis en 2 tiempos quirúrgicos, utilizando en todos los casos, cuando es posible, el nervio maseterino como nervio donador y dejando un intervalo de entre $4 \mathrm{y}$ 6 meses entre una cirugía y otra. ${ }^{(10)}$ Lischez, por su parte, recoge su experiencia en 2 casos dejando un periodo de 1 año entre una cirugía y otra. ${ }^{(14)}$

Los únicos casos en la literatura que presentan restauración dinámica de la sonrisa en pacientes con Síndrome de Möbius completo en un solo tiempo quirúrgico son los de Woollard,${ }^{(8)}$ quien utilizó la rama maseterina como nervio donador para inervar colgajos de dorsal ancho segmentarios bilaterales en 20 pacientes, y Chuang ${ }^{(15)}$ en 2013, quien publicó su experiencia en 6 pacientes en los que utilizó como nervio donador el espinal para inervar el músculo gracilis transferido de forma bilateral en un solo tiempo quirúrgico, presentado en sus paciente inicio de movimientos a los 3 meses, hasta lograr una sonrisa independiente a los 6 meses en niños y al año en adultos.

En nuestro estudio llevamos a cabo la reanimación dinámica facial en un solo tiempo quirúrgico en 4 pacien- 
tes con Síndrome de Möbius completo, utilizando en 2 casos la rama maseterina y en otros 2 el nervio espinal como nervios donadores. Desde entonces hemos realizado la reanimación facial bilateral en nuestros pacientes en un solo tiempo quirúrgico debido a su baja morbilidad, el corto tiempo de cirugía (el promedio de la duración total del procedimiento fue de 6.5 horas, con un máximo de 8 horas y un mínimo de 5 horas, mejorando progresivamente nuestros tiempos a medida que ganamos experiencia), y a que hemos logrado el inicio de movimientos en los músculos trasferidos alrededor de los 3 meses de la intervención y la contracción muscular completa a los 11 meses de la cirugía. El resto de nuestros pacientes operados, 2 pacientes más en total a partir de octubre del 2016, aún se encuentran en etapa de seguimiento.

En cuanto a los nervios donadores, no existe consenso respecto a cuál es el mejor nervio para neurotizar el obturador. La literatura al respecto refiere el uso del nervio facial contralateral, en casos de Möbius incompleto o Möbius-Like, nervio hipogloso, rama maseterina del trigémino, rama motora de $\mathrm{C} 7$, ramas motoras cervicales, nervio frénico y nervio espinal accesorio. En nuestra experiencia, los mejores resultados los hemos obtenido utilizando el nervio facial contralateral, cuando se encuentra disponible, la rama maseterina y el nervio espinal accesorio, siendo la rama maseterina nuestra primera elección en pacientes con Síndrome de Möbius completo.

En la serie de 4 pacientes que presentamos utilizamos la rama maseterina como nervio donador en 2 de ellos, y en el resto el nervio espinal accesorio; esto se debió a que en a la exploración física inicial apreciamos una disminución en la fuerza de la mordida y posteriormente la electromiografía preoperatoria confirmó una disminución en la actividad motora del masetero. Hasta el momento, con estos nervios donadores, hemos logrado una contracción completa en todos los casos. Sin embargo, en aquellos casos en los que utilizamos el nervio espinal notamos mejores calificaciones en las escalas utilizadas para evaluación de resultados; pero cabe señalar que estos casos también coinciden con los de mayor tiempo de postoperatorio, por lo que habrá que continuar con el seguimiento para poder comparar ambos grupos.

En la evolución posterior que hemos podido recoger y a medida que realizamos más cirugías, hemos modificado nuestras preferencias de elección de nervio donador por el nervio espinal debido a los mejores resultados que hemos obtenido hasta el momento en todas las escalas aplicadas; sin embargo la recomendación final podrá realizarse una vez que completemos el seguimiento mínimo de rehabilitación de 30 meses para ambos grupos.

Ningún paciente de nuestra serie presentó complicaciones, manteniendo una fuerza de mordida adecuada y de elevación de hombros, sin dolor de cuello, hombros o al realizar mordida.

\section{Conclusiones}

La cirugía de reanimación facial mediante transferencia de colgajo libre de gracilis bilateral en un solo tiempo quirúrgico en pacientes con Síndrome de Möebius completo es un procedimiento seguro y efectivo para el tratamiento de la parálisis facial bilateral que presentan estos pacientes. Es indispensable realizar una selección adecuada de los pacientes, analizar cada caso de forma independiente mediante exploración física y electromiografía preoperatorias, llevar a cabo una cirugía impecable, con un adecuado manejo de los tejidos, y posteriormente propiciar una rehabilitación completa de la sonrisa. Todos estos son factores muy importantes que nos llevarán al éxito en la reconstrucción facial de este grupo de pacientes.

Con la experiencia que hemos desarrollado al tratar estos casos sugerimos utilizar el nervio espinal accesorio como primera opción de nervio donador en la neurotización de los colgajos libres y dejar como segunda opción la rama maseterina del trigémino.

\section{Dirección del autor}

Dr. Alexandro Aguilera Salgado

División de Cirugía Plástica

Instituto Nacional de Pediatría

Insurgentes Cuicuilco, 3700, Letra C, Coyoacán 04530.

Ciudad de México, México

Correo electrónico: dr.alexandroaguilera@gmail.com

\section{Bibliografía}

1. Terzis JK, Noah EM. Dynamic restoration in Möbius and Möbius-like patients. Plast Reconstr Surg. 2003;111:40-55.

2. Camacho-Ramírez RI, Ávila-Reyes Ricardo, et al. Caso Clínico: Síndrome de Moebius. Archivos de Investigación Pediátrica de México 2007; 10(1): 16-20.

3. Gómez-Valencia L, Morales Hernández A, et al. Estudio Clínico y Genético del Síndrome de Moebius. Bol Med Hosp Infant Mex 2008; 65: 353-357.

4. Carrillo-Hernández CA, Romo Chávez H. Síndrome de Moebius. Revista de Especialidades Médico-Quirúrgicas 2010; 15(4): 261-265.

5. Palafox D, Arrieta-Joffe P, Cárdenas-Mejía A. Tratamiento quirúrgico reconstructivo actual del síndrome de Moebius. $\mathrm{Ci}$ rugía Plástica. 2014; 24 (3): 136-144.

6. Terzis J. Moebius and Moebius-like patients: etiology, diagnosis, and treatment options. Clin Plastic Surg 29 (2002) 497514.

7. Singham J, Manktelow R, Zuker RM. Möbius syndrome. Semin Plast Surg. 2004; 18 (1): 39-45.

8. Woollard AC, Harrison DH, Grobbelaar AO. An approach to bilateral facial paralysis. J Plast Reconstr Aesthet Surg. 2010;63:1557-1560.

9. Terzis JL, Olivares FS. Long-term outcomes of free muscle transfer for smile restoration in children. Plast Reconstr Surg. 2009; 123 (2): 543-555.

10. Zuker RM, Goldberg CS, Manktelow RT. Facial animation in children with Mobius syndrome after segmental gracilis muscle transplant. Plast Reconstr Surg. 2000;106:1-8. 
11. Chuang DC. Free tissue transfer for the treatment of facial paralysis. Facial Plast Surg. 2008;24:194-203.

12. Chuang DC. Technique evolution for facial paralysis reconstruction using functioning free muscle transplantation in Chang Gung Memorial Hospital. Clin Plast Surg. 2002;29:449-459.

13. Janakie S, Manktelow R, Zuker R. Moebius Syndrome. Seminars in Plastic Surgery. 2004; 18 (1): 176-185.

14. Lischez SD, Matloub HS, Gosain AK. Cortical adaptation to restoration of smiling following free muscle transfer innervated by the nerve to the masseter. Plast Reconstr Surg. 2005; 115:1472-1479.
15. Chuieng-Yi Lu, Chwei-Chin Chuang. One-Stage Reconstruction for Bilateral Moebius Syndrome. Simultaneous Use of Bilateral Spinal Accessory Nerves to Innervate 2 Free Muscles for Facial Reanimation. Annals of Plastic Surgery. 2013.70 (2): 1011-1021.

16. Arrieta Joffe, P. y col. Estudio clínico, citogenético, molecular y de imagen de los pacientes con Síndrome de Moebius del Hospital General "Dr. Manuel Gea González", Ciudad de México. Cir plast, iberolatinoam. 2017, 43(4): 395-400.

\title{
Comentario al artículo “Reanimación facial en Síndrome de Möhius completo mediante transferencia en un solo tiempo de gracilis libre bilateral"
}

\author{
Bernardo HONTANILLA-CALATAYUD \\ Cirujano Plástico, Director del Departamento de Cirugía Plástica, Estética y Reparadora, Clínica Universidad de Navarra. \\ Catedrático Cirugía Plástica, Universidad de Navarra. Pamplona, España.
}

En primer lugar felicitar a los autores por el esfuerzo que supone realizar dos trasplantes musculares libres en un solo tiempo quirúrgico en la reconstrucción de la sonrisa del Síndrome de Moebius. Por otro lado, quería hacer algunos comentarios constructivos al artículo publicado.

$\mathrm{Al}$ igual que los autores, pienso que el nervio masetérico es la mejor fuente nerviosa donante en este tipo de pacientes. Sin embargo, donde no coincido es en la utilización del nervio espinal como segunda opción, a no ser que el nervio hipogloso esté afectado en el síndrome ${ }^{(1)}$ Hay que tener en cuenta que no sólo se trata de conseguir un movimiento facial sino que también este movimiento tiene que ser espontáneo y disociado del nervio donante. Así, las posibilidades de conseguir un movimiento espontáneo son mayores cuando la representación del movimiento en la corteza cerebral motora está más próxima a la corteza motora del nervio facial. (2)

En cuanto a los resultados obtenidos, sería preferible ver vídeos pre y postcirugía para valorar el resultado. Se dan casos de Síndrome de Moebius que presentan un leve movimiento de un lado de la cara y que al hacer un solo trasplante, se desarrolla el movimiento del lado no operado. Si sólo se aportan fotos se corre el peligro de sesgar el resultado. En el caso primero, aparentemente se aprecia una tracción leve de las comisuras que no se corresponde con la buena tracción que habitualmente se obtiene con este nervio masetérico y que está abundantemente publicada en la literatura científica. Más aun, la tracción parece ejercerse por detrás del surco nasogeniano que puede haber sido por colocación del músculo por detrás del surco o porque este se haya desplazado a posteriori. En el segundo caso, la tracción es mayor utilizando el nervio espinal. Cabe la duda, es opinión personal, de si en vez de utilizar dos puntos en la coaptación nerviosa y un pegamento biológico se hubiera conseguido mejor tracción si se hubieran coaptado los nervios completamente con puntos sueltos sin la utilización del pegamento.

En cualquier caso, como bien dicen los autores, es necesario un mayor tiempo de seguimiento para detectar la disociación y espontaneidad sobre todo cuando se utiliza el nervio espinal, siendo este movimiento espontáneo el componente más importante de la sonrisa.

Bibliografía

1. Hontanilla B, Cabello A. J. Spontaneity of smile after facial paralysis rehabilitation when using a non-facial donor nerve. Craniomaxillofac Surg. 2016;44(9):1305-1309.

2. Buendia J, Loayza FR, Luis EO, Celorrio M, Pastor MA, Hontanilla B.J. Functional and anatomical basis for brain plasticity in facial palsy rehabilitation using the masseteric nerve. Plast Reconstr Aesthet Surg. 2016;69(3):417-426. 


\section{Respuesta al comentario del Dr. B. Hontanilla-Calatayud}

\section{Alexandro AGUILERA-SALGADO}

Agradecemos al Dr. Hontanilla sus amables y constructivos comentarios acerca del trabajo que hemos realizado al lograr en nuestros pacientes la reanimación facial en ambos lados de la cara en un solo tiempo quirúrgico.

En cuanto a la selección del nervio donante, está descrito el uso de diversos nervios cercanos, logrando distintos grados de fuerza y de espontaneidad. El Dr. David Chuang describe las características que debe tener un nervio motor ideal para inervar el músculo libre utilizado en reanimación facial: nervio cercano que no requiera injerto nervioso; nervio fuerte que otorgue inervación adecuada; tiempo de rehabilitación corto; mínimas morbilidades; potencial de lograr sonrisa espontánea; y aplicación universal en diversas situaciones. ${ }^{(1)}$

Como mencionamos, en nuestra práctica diaria es rutinaria la selección del nervio maseterino como primera opción en los pacientes en quienes está disponible. En aquellos en los que está afectado, optamos por utilizar el nervio espinal por diversas razones: no necesitamos injerto nervioso; fácil disección; por la edad de los pacientes (4 y 6 años) la plasticidad cerebral permite adaptabilidad en la representación cortical, logrando movimientos espontáneos; nervio fuerte que permite reinervación adecuada; produce mínima morbilidad en el sitio donador; y el tiempo de rehabilitación es corto. No consideramos el uso del nervio hipogloso debido a que, al tratarse de una reanimación facial bilateral, la morbilidad en el sitio donador puede ser muy importante, pudiendo ocasionar dificultad para el lenguaje o la deglución. En caso de utilizar un porcentaje menor del nervio para evitar estas complicaciones potenciales $(40 \%)$, consideramos que la fuerza de reinervación para lograr una contracción fuerte del músculo trasplantado será menor, con resultados menos favorables.
El uso del nervio espinal está descrito por diversos autores con resultados excelentes, como Chuang y Terzis, logrando fuerzas de contracción completas y espontáneas, tal y como hemos logrado replicar en nuestros casos. ${ }^{(1)}$

De igual forma, en nuestra práctica diaria realizamos las coaptaciones nerviosas con puntos simples de nylon 10-0 y posteriormente gel de fibrina. Se han publicado en la literatura diversos trabajos en los que no existe diferencia significativa en cuanto al uso de sutura o pegamento, siempre y cuando se logre una coaptación eficiente, sin tensión y con buen afrontamiento entre ambos cabos de los nervios, por lo que no consideramos que nuestra técnica de coaptación haya influido en nuestros resultados. ${ }^{(2)}$ En los videos que, a petición del Dr. Hontanilla adjuntamos y que están disponibles en la edición electrónica de la revista, la contracción se puede apreciar adecuadamente. La paciente a la cual hace referencia es adulta y en ella utilizamos como donante el nervio maseterino.

Continuamos trabajando en estos casos y, más adelante, compartiremos la evolución de nuestros pacientes con un seguimiento a largo plazo.

Bibliografía

1. Chuang DC. One-Stage Procedure Using Spinal Accessory Nerve (XI)-Innervated Free Muscle for Facial Paralysis Reconstruction. Plast Reconstr Surg. 2013; 132 (1): 117-129.

2. Sameem M. A Systematic Review on the Use of Fibrin Glue for Peripheral Nerve Repair. Plast. Reconstr. Surg. 2011, 127: 2381-2390.

Video complementario al artículo en: www.ciplaslatin.com 